\title{
Sudden Intraoperative Hyperkalemia during Laparoscopic Radical Nephrectomy in a Patient with Underlying Renal Insufficiency
}

\author{
Sung Hoon Jung, Yun-Joung Han, Sang Ho Shin, Hyo Seon Lee, Ji Young Lee \\ Department of Anesthesiology and Pain Medicine, Yeouido St. Mary's Hospital, The Catholic University of Korea, Seoul, Korea
}

We experienced a case of severe intraoperative hyperkalemia during laparoscopic radical nephrectomy in a 60-year-old male patient with renal insufficiency, whose hypertension had been managed by preoperative angiotensin II receptor blocker (ARB) and adrenergic beta-antagonist. After renal vessel ligation, his intraoperative potassium concentration suddenly increased to $7.0 \mathrm{mEq} / \mathrm{L}$, but his electrocardiography (ECG) did not show any significant change. While preoperative ARB therapy has been regarded as a contributing factor for further aggravation of underlying renal insufficiency, we assumed that nephrectomy itself and rhabdomyolysis caused by surgical trauma also aggravated the underlying renal dysfunction and resulted in sudden hyperkalemia. Hyperkalemia was managed successfully with calcium gluconate, insulin, furosemide and crystalloid loading during the intraoperative and immediate postoperative periods, and potassium concentration decreased to $5.0 \mathrm{mEq} / \mathrm{L}$ at 8 hours after the operation. The patient's hospital course was uncomplicated, but his renal function deteriorated further.

Key Words: adrenergic beta-antagonist; angiotensin II receptor blocker; hyperkalemia; renal insufficiency; rhabdomyolysis

In patients who have risk factors predisposing to hyperkalemia, the possibility of hyperkalemia has to be considered. The underlying renal dysfunction and the medication known to increase serum potassium concentration are the most commonly known factors in patients with hyperkalemia. Also, drug therapy or underlying renal insufficiency were the leading causes of hyperkalemia among inpatients [1-3]. Surgery associated with muscle damage, high-volume blood transfusion, and various medications are the most frequent causes of hyperkalemia [4]. In many patients, the cause of hyperkalemia is multifactorial and difficult to define clearly. Here in this case report, we present a case of severe intraoperative hyperkalemia due to multiple causative factors without any notable ECG abnormality, which was treated during the postoperative period.

\section{CASE REPORT}

A 60-year-old male patient underwent laparoscopic radical nephrectomy for a 6.3-cm-sized solid mass in the lower pole of the left kidney, under general anesthesia. His body parameters

\section{Case Report}

Received: August 8, 2016

Revised: October 13, 2016

Accepted: October 14, 2016

Corresponding author

Ji Young Lee

Department of Anesthesiology and

Pain Medicine, Yeouido St. Mary's

Hospital, The Catholic University

of Korea, 10 63(yuksam)-ro,

Yeongdeungpo-gu, Seoul 07345,

Korea

Tel: +82-2-3779-1944

Fax: +82-0303-3440-1268

E-mail: leeji@catholic.ac.kr

Copyright (@) 2018 The Korean Society of Critical Care Medicine

This is an Open Access article distributed under the terms of Creative Attributions Non-Commercial License (http:// creativecommons.org/li-censes/by-nc/4.0/ which permits unrestricted noncommercial use, distribution, and reproduction in any medium, provided the original work is properly cited. 
were weight $84.2 \mathrm{~kg}$, height $175.3 \mathrm{~cm}$, and body mass index $27.4 \mathrm{~kg} / \mathrm{m}^{2}$. He had hypertension and diabetes for 20 years, gout for 10 years, and dyslipidemia for an unknown period. Each disease was treated with daily medication including ARB valsartan 80 mg (Diovan; Novartis Pharma Schweiz, Rotkreuz, the Switzerland), adrenergic beta-antagonist carvedilol 25 mg (Dilatrend; Chong Kun Dang Pharmaceutical Corp., Seoul, Korea), calcium channel blocker amlodipine $5 \mathrm{mg}$ (Orodipine; Dong-A ST, Seoul, Korea), oral antidiabetic agent glimepiride $25 \mathrm{mg}$ (Glimel, Dong-A ST), and xanthine oxidase inhibitor allopurinol 100 mg (Zyloric; Samil Co., Seoul, Korea). He had been a smoker and had a drinking history. On radiologic evaluation, both kidneys of the patient were filled with multiple cysts in addition to the mass in the lower pole of the left kidney (Figure 1).

With respect to his laboratory values, parameters indicating renal function were moderately decreased to levels found in

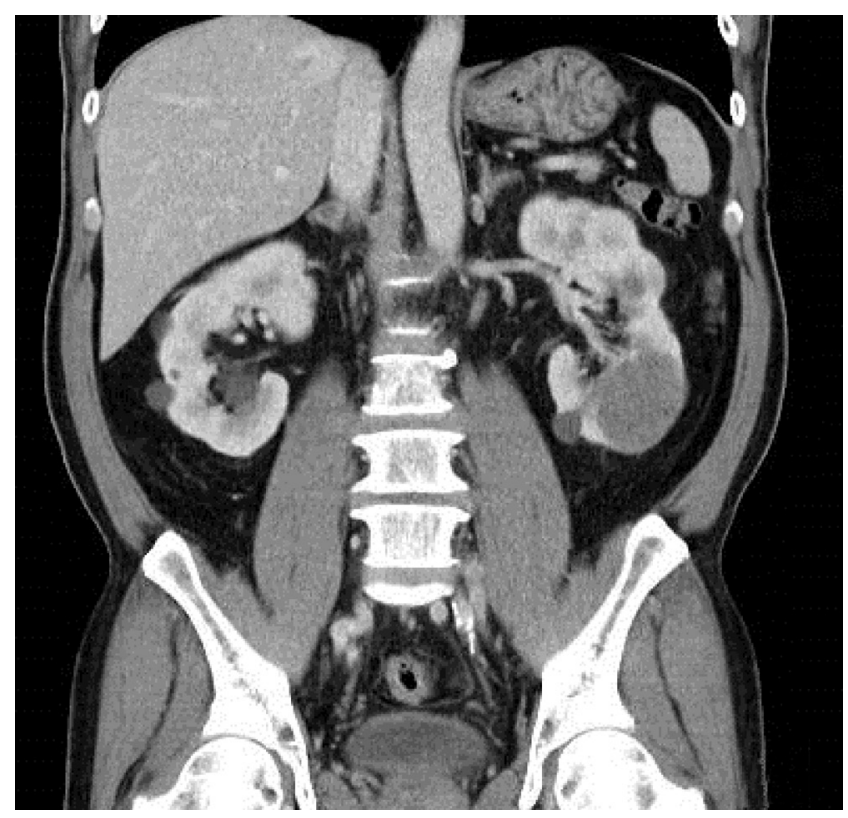

Figure 1. Abdominal and pelvic spiral computed tomography shows an approximately $6.3-\mathrm{cm}$-sized probable enhancing solid lesion in the lower pole of the left kidney and multiple variable-sized cysts in both kidneys. the chronic kidney disease 3a stage, as shown below: blood urea nitrogen $23.9 \mathrm{mg} / \mathrm{dl}$, serum creatinine $1.3 \mathrm{mg} / \mathrm{dl}$, and estimated glomerular filtration rate (eGFR) $57.9 \mathrm{ml} / \mathrm{min} / 1.73 \mathrm{~m}^{2}$. Also, hemoglobin Alc was increased to $7.9 \%$. His blood glucose values were maintained in the range of $174-194 \mathrm{mg} / \mathrm{dl}$ preoperatively. His ECG showed left ventricular hypertrophy.

We induced general anesthesia with propofol $150 \mathrm{mg}$, supplementary remifentanil infusion at a rate of $0.2 \mu \mathrm{g} / \mathrm{kg} / \mathrm{min}$, rocuronium bromide $50 \mathrm{mg}$, and intubation with an endotracheal tube with an inner diameter of $8.0 \mathrm{~mm}$, and we maintained anesthesia with desflurane 6 vol\%-air-oxygen mixture with $\mathrm{FiO}_{2}$ 0.4. The operation was performed in a right down, semi-lateral $\left(30^{\circ}\right)$, and fully flexed decubitus position. We monitored the patient's physiologic parameters including ECG lead II, noninvasive blood pressure, end-tidal carbon dioxide concentration, central venous pressure (CVP), direct arterial pressure through the right radial arterial catheter, and the arterial blood gas analysis result.

Two hours and thirty minutes from the start of the operation, the vessels and the ureter of the left kidney were ligated successfully. We performed the second arterial blood gas analysis and found severe hyperkalemia with a potassium concentration of $7.0 \mathrm{mEq} / \mathrm{L}$ and mild hyponatremia with a sodium concentration of $129.7 \mathrm{mEq} / \mathrm{L}$. Even though there was no change in the ECG, we infused 2 gm of calcium gluconate over 3 minutes, initially. Also, we started to infuse the mixture of regular insulin (RI) $20 \mathrm{IU}$ and potassium chloride $10 \mathrm{mEq}$ in $500 \mathrm{ml}$ of normal saline, at a rate of $40 \mathrm{ml} / \mathrm{hr}$. The crystalloid solutions were changed from Hartmann's solution to normal saline in order to avoid potassium load. We injected furosemide $20 \mathrm{mg}$ and another dose of $20 \mathrm{mg}$ at an interval of 20 minutes to increase the urine output. After 40 minutes from the detection of hyperkalemia and prompt treatment, the serum sodium/ potassium value was 129.6/6.3 mEq/L (Table 1). We extubated the trachea after complete reversal of neuromuscular blockade with sugammadex $200 \mathrm{mg}$. Total anesthesia duration was 4 hours 50 minutes. Total amount of fluid infused was 1,950 $\mathrm{ml}$ of crystalloids and total fluid loss was approximately 350

Table 1. The changes in serum sodium and potassium levels during and after the operation

\begin{tabular}{|c|c|c|c|c|c|c|c|c|}
\hline Variable & $\begin{array}{l}\text { Start of the } \\
\text { operation }\end{array}$ & $\begin{array}{c}\text { After renal } \\
\text { vessel ligation }\end{array}$ & $\begin{array}{c}40 \text { Minutes after } \\
\text { hyperkalemia } \\
\text { treatment }\end{array}$ & $\begin{array}{l}\text { End of the } \\
\text { operation }\end{array}$ & PACU & ICU arrival & $\begin{array}{l}8 \text { Hours } \\
\text { after the } \\
\text { operation }\end{array}$ & POD 1 \\
\hline \multicolumn{9}{|c|}{ Electrolyte (normal value, mEq/L) } \\
\hline Sodium (135.0-148.0) & 133.0 & 129.7 & 129.6 & 130.5 & 130.0 & 132.0 & 136.0 & 138.0 \\
\hline Potassium (3.5-5.3) & 4.8 & 7.0 & 6.3 & 6.7 & 5.5 & 5.2 & 5.0 & 5.0 \\
\hline
\end{tabular}

PACU: postanesthesia care unit; ICU: intensive care unit; POD: postoperative day. 
$\mathrm{ml}$ (urine output $250 \mathrm{ml}$ and estimated blood loss $100 \mathrm{ml}$ ). In the postanesthesia care unit (PACU), serum sodium/potassium values were 130.5/6.7 mEq/L on arrival and 130.0/5.5 $\mathrm{mEq} / \mathrm{L}$ before leaving the PACU. The patient was moved to the surgical intensive care unit (ICU).

In the surgical ICU, we used the CVP value and urine output to guide fluid management. The average amount of fluid administered in the ICU was 3,404 $\mathrm{ml} /$ day and the urine output was 2,496 ml/day (104.0 ml/hr). Diuretics were used immediately, furosemide $20 \mathrm{mg}$ on the 2 nd postoperative day. We analyzed serum myoglobin and creatine kinase levels postoperatively on the day of the operation, under suspicion of rhabdomyolysis. The serum myoglobin level was $648 \mathrm{ng} / \mathrm{ml}$ (reference value, $<70 \mathrm{ng} / \mathrm{ml}$ ) and the creatine kinase level was $285 \mathrm{IU}$ (reference value, 0 to $171 \mathrm{IU}$ ). The serum potassium level decreased to the normal range at 8 hours after the end of anesthesia. He stayed in the ICU for 2 days and was discharged 11 days after the operation. His serum potassium concentrations were maintained in the range of $4.6-5.0 \mathrm{mEq} / \mathrm{L}$ until discharge.

\section{DISCUSSION}

The incidence of hyperkalemia has been reported to be one to 10 per 100 inpatients. This wide range of incidence was due to the wide range of inclusion threshold. In the study by Acker et al. [1], the inclusion threshold for hyperkalemia was a serum potassium level above $6.0 \mathrm{mEq} / \mathrm{L}$, while the inclusion threshold for hyperkalemia was a serum potassium level above 5.3 $\mathrm{mEq} / \mathrm{L}$ in the report by Shemer et al. [5]. Recently, the incidence of hyperkalemia (serum potassium level $>5.0 \mathrm{mEq} / \mathrm{L}$ ) in hospitalized patients was reported to be $7.3 \%$ [2]. When we care for surgical patients as a critical care clinician, we should be aware of the causes of pre-, intra-, and postoperative hyperkalemia, on the basis of the reported incidence of hyperkalemia.

In this case report, the patient had two major preoperative factors of hyperkalemia. These were decreased renal function and drugs that can increase the serum potassium level. There were multiple differently sized cysts in both kidneys of the patient, reducing functionally active parenchyma. Also, hypertension, diabetes, and gout might have contributed to renal function impairment in this patient. His laboratory values indicating renal function were abnormal.

Hyperkalemia was detected during the period between complete renal vessel ligation and removal of the kidney from the abdomen. To suggest that there was a time interval from renal artery clamping to detection of hyperkalemia, loss of whole left kidney function should have contributed to the development of hyperkalemia. Because both kidneys of the patient had multiple cysts, we could not determine which kidney was functionally dominant. The patient's eGFR value decreased to one-half of the preoperative value after surgery. We supposed that the preoperative function of both kidneys was similarly decreased, and sudden reduction of functioning parenchyma to one-half after left renal artery clamping contributed to decreased potassium excretion.

Nonrenal factors, endocrinal factors, and medications can contribute to the development of hyperkalemia. These factors can disturb the renin-angiotensin-aldosterone system (RAAS), the main hormonal system that regulates renal potassium excretion. Patients with chronic renal disease, most often diabetic nephropathy, may have hyporeninemic hypoaldosteronism [6]. Some drugs that cause RAAS blockade may predispose to hyperkalemia. They include angiotensin converting enzyme inhibitors (ACEi), ARBs, direct renin inhibitors, nonsteroidal anti-inflammatory drugs, and adrenergic beta-antagonists [7] ACEi/ARB cause hyperkalemia via several mechanisms including decreased aldosterone concentrations, decreased delivery of sodium to the distal nephron, abnormal collecting tubule function, and excessive potassium intake [8]. Among inpatients taking ARBs, the patient-month of hyperkalemia was reported to be $5.4 \%$ during the study period, and moderate to fatal hyperkalemia was relatively rare [9]. On the other hand, adrenergic beta-antagonists suppress renin release which is stimulated by catecholamine and decrease aldosterone synthesis, ultimately leading to hyperkalemia [10]. The patient in this case report took valsartan which is an ARB, and carvedilol, on adrenergic beta-antagonist. Both drugs seemed to contribute to the development of hyperkalemia. We were advised to exercise caution while using aldosterone blockade in people with advanced stage 3 nephropathy who have a serum potassium level of $4.5 \mathrm{mEq} / \mathrm{L}$ for safety from hyperkalemia [11].

During surgery, potassium may be released from damaged cells as a result of intraoperative tissue damage, ischemia, and rhabdomyolysis. The most common cause of rhabdomyolysis is muscular trauma, and surgery performed in an improper position predisposes to the development of rhabdomyolysis. Rhabdomyolysis after nephrectomy in the lateral decubitus position has been rarely reported $[12,13]$. A lateral decubitus position, especially a full-flexed flank position for nephrectomy, long operative time, increased body mass index, and extracellular volume depletion are the predisposing factors of rhabdomyolysis after surgery. Additional factors are male sex, 
younger age, diabetes, chronic kidney disease, and perioperative bleeding. Many factors mentioned above were observed in the patient presented in this case report. Even though the degree of rhabdomyolysis was not severe based on slightly elevated serum creatine kinase and myoglobin levels, rhabdomyolysis itself might contribute to the development of hyperkalemia.

While the diagnostic criteria and treatment of hyperkalemia are well known, confirming the diagnosis of hyperkalemia is not easy. Patients with severe hyperkalemia frequently have normal or abnormal ECG, but these are inconsistent with hyperkalemia. Among patients with potassium levels greater than $6.0 \mathrm{mEq} / \mathrm{L}$, only $46 \%$ showed ECG changes. Also, only $55 \%$ of the patients with potassium levels greater than $6.8 \mathrm{mEq} / \mathrm{L}$ showed changes consistent with hyperkalemia [1]. The reason for challenges in the diagnosis of hyperkalemia is that neither the ECG nor the serum potassium level alone is an adequate index of the urgency of hyperkalemia [14]. In hyperkalemia, the ECG manifestations are generally progressive and proportional to the serum potassium level. But, ventricular fibrillation may be the first ECG abnormality of hyperkalemia; conversely, a normal ECG may be seen in patients with severe hyperkalemia. The patient in this case report did not show any ECG abnormalities to make us doubt the possibility of pseudohyperkalemia. This condition was ruled out after repeated cautious laboratory tests during treatment. It is apparent that we must consider the clinical context while evaluating hyperkalemic patients because neither the ECG nor the serum potassium level alone is an adequate index of the urgency of hyperkalemia.

The recommended guidelines for emergent treatment of hyperkalemia are as follows: (1) serum potassium level greater than $6.5 \mathrm{mEq} / \mathrm{L}$ and (2) ECG manifestations of hyperkalemia regardless of the serum potassium level. On this reasonable basis, we treated hyperkalemia through membrane stabilization (calcium gluconate), redistribution (temporary hyperventilation and insulin), and elimination (furosemide). We mixed RI in normal saline instead of dextrose solution because the patient's blood sugar level was too high. It has been recommended to use insulin without dextrose in hyperglycemic patients [15].

In conclusion, our experience of sudden intraoperative hyperkalemia without ECG changes in a patient who underwent laparoscopic radical nephrectomy reminds us of the possible causes of sudden hyperkalemia. Pre-existing renal insufficiency, preoperative antihypertensive medications including ACEi, ARB, and adrenergic beta-antagonist, and surgery-related fac- tors such as rhabdomyolysis can increase the risk of hyperkalemia.

\section{CONFLICT OF INTEREST}

No potential conflict of interest relevant to this article was reported.

\section{ORCID}

Ji Young Lee https://orcid.org/0000-0001-9123-1135

\section{REFERENCES}

1. Acker CG, Johnson JP, Palevsky PM, Greenberg A. Hyperkalemia in hospitalized patients: causes, adequacy of treatment, and results of an attempt to improve physician compliance with published therapy guidelines. Arch Intern Med 1998;158: 917-24.

2. Eliacik E, Yildirim T, Sahin U, Kizilarslanoglu C, Tapan U, Aybal-Kutlugun A, et al. Potassium abnormalities in current clinical practice: frequency, causes, severity and management. Med Princ Pract 2015;24:271-5.

3. Rimmer JM, Horn JF, Gennari FJ. Hyperkalemia as a complication of drug therapy. Arch Intern Med 1987;147:867-9.

4. Ayach T, Nappo RW, Paugh-Miller JL, Ross EA. Postoperative hyperkalemia. Eur J Intern Med 2015;26:106-11.

5. Shemer J, Modan M, Ezra D, Cabili S. Incidence of hyperkalemia in hospitalized patients. Isr J Med Sci 1983;19:659-61.

6. Grande Villoria J, Macias Nunez JF, Miralles JM, De Castro del Pozo S, Tabernero Romo JM. Hyporeninemic hypoaldosteronism in diabetic patients with chronic renal failure. Am J Nephrol 1988;8:127-37.

7. Schaefer TJ, Wolford RW. Disorders of potassium. Emerg Med Clin North Am 2005;23:723-47.

8. Raebel MA. Hyperkalemia associated with use of angiotensin-converting enzyme inhibitors and angiotensin receptor blockers. Cardiovasc Ther 2012;30:e156-66.

9. Park I, Sheen SS, Lim HS, Yoon D, Park MY, Lee SH, et al. Comparison of hyperkalemic risk in hospitalized patients treated with different angiotensin receptor blockers: a retrospective cohort study using a Korean clinical research database. Am J Cardiovasc Drugs 2012;12:255-62.

10. Hahn L, Hahn M. Carvedilol-induced hyperkalemia in a patient with chronic kidney disease. J Pharm Pract 2015;28:107.

11. Khosla N, Kalaitzidis R, Bakris GL. Predictors of hyperkalemia risk following hypertension control with aldosterone block- 
ade. Am J Nephrol 2009;30:418-24.

12. van Dellen D, Tavakoli A, Wadsworth R, Augustine T. Intraoperative hyperkaliemia complicating hand-assisted live-donor nephrectomy. Exp Clin Transplant 2011;9:417-20.

13. Pariser JJ, Pearce SM, Patel SG, Anderson BB, Packiam VT, Shalhav AL, et al. Rhabdomyolysis after major urologic surgery: epidemiology, risk factors, and outcomes. Urology 2015; 85:1328-32.
14. Parham WA, Mehdirad AA, Biermann KM, Fredman CS. Hyperkalemia revisited. Tex Heart Inst J 2006;33:40-7.

15. Kline JA, Weisberg LS. Acid-base, electrolyte, and metabolic abnormalities. In: Parrillo JE, Dellinger RP, editors. Critical care medicine: principles of diagnosis and management in the adult. 4th ed. Philadelphia: Elsevier Saunders; 2014. p. 993-1028. 